\title{
Vergleichende Untersuchung der Holzqualität gesunder und kranker Fichten und Tannen von 6 schweizerischen Standorten
}

\author{
J. Sell, G.Schnell und M. Arnold \\ Abteilung Holz der EMPA, Dübendorf/Schweiz*
}

\begin{abstract}
Insgesamt kann mit aller Eindeutigkeit festgestellt werden, da $\beta$ das Holz geschädigter Fichten und Tannen gegenüber gesunden Bäumen der gleichen Herkunft keine Qualitätsverminderung aufweist und deshalb uneingeschränkt verwendbar ist; lediglich bei der Auswahl von Rundholz für die Mastenimprägnierung sollte darauf geachtet werden, daß die Splintbreite nicht zu gering ist. Geringe Eigenschaftsunterschiede des Holzes der (insgesamt 235) untersuchten Bäume lassen sich zumindest teilweise aus langfristigen Unterschieden ihrer sozialen Stellung und anderer Wachstumsbedingungen erklären.
\end{abstract}

\section{Wood quality of healthy and diseased spruce and fir (Picea abies and Abies alba) \\ from 6 different Swiss locations}

It can be stated clearly that wood of diseased spruces and firs - compared with healthy trees of identical stands - does not exhibit a reduction of its quality and, therefore, can be used without restrictions. Only when roundwood is to be selected for preservative-treated poles one should pay attention to a normal sapwood width. Little differences of the properties of the (totally 235) trees investigated can at least partially be explained by their social position within the forest stands and by other growth conditions.

\section{Einleitung}

Da der gesicherte Absatz des Holzes von erkrankten Bäumen für die Wald- und Holzwirtschaft von erheblicher Bedeutung ist, muß durch vielfältige $\mathrm{Maßnahmen}$ auch in der Schweiz sichergestellt werden, daß keine unbegründeten Zweifel an der Qualität des Holzes solcher Bäume bestehen bleiben. Die vorliegende Untersuchung im Rahmen der mehrjährigen schweizerischen Waldschadenserhebung Sanasilva, Teilprojekt „Holzqualität", hatte das Ziel, einen Vergleich des Holzes von Nadelbäumen verschiedener Schädigungsstufen über ein möglichst breites, praxisbezogenes Qualitätsspektrum durchzuführen. Der Schwerpunkt war dabei auf die Eigenschaften des Baustoffes Holz zu legen, da der Baumarkt mit annähernd $50 \%$ des Holzabsatzes der wichtigste Marktsektor für Holzprodukte ist.

\footnotetext{
* Mitarbeit von E. Graf, Abt. Biologie der EMPA St. Gallen (Biologische Resistenz und Imprägnierbarkeit), sowie K. Richter, Abt. Holz der EMPA Dübendorf (Jahrringanalyse)
}

\section{Bisherige Kenntnisse}

Seit sich in den späten siebziger Jahren, vor allem aber seit 1983/84, in Mitteleuropa (besonders in Deutschland, Österreich und in der Schweiz) die Meldungen über ungewöhnlich intensive Schäden an Waldbäumen häuften, hat eine beispiellose Forschungsaktivität über Umfang, Ursachen und Auswirkungen dieser Schäden eingesetzt. Es wird hierzu auf die laufend aktualisierten Bibliographien verwiesen, z. B. auf die von der Bundesanstalt für Forst- und Holzwirtschaft Hamburg herausgegebene Literaturzusammenstellung [Schrader et al. 1983-1986]. Allein schon das enge Fachgebiet "Baumschäden und Holzqualität" ist so umfangreich dokumentiert, daß hier nur eine sehr summarische Wiedergabe erfolgen kann. Ein Überblick über die bisherigen deutschen Forschungsarbeiten auf diesem Gebiet ist auf einer Arbeitstagung des Deutschen Waldschadensausschusses, Bereich Holzqualität, gegeben worden; kürzlich haben Schulz et al. (1988) eine weitere Zusammenfassung der deutschen Forschungsarbeiten veröffentlicht. Aus den bislang vorliegenden Ergebnissen dieser z. T. noch laufenden Vorhaben läßt sich im Hinblick auf die Qualität des Holzes folgendes eindeutig feststellen (vgl. Sell et al. 1988):

Chemische Zusammensetzung des Holzes: kein Unterschied von praktischer Bedeutung.

Holzanatomische Merkmale: überwiegend keine signifikant verschiedenen Ergebnisse zwischen gesunden und geschädigten Bäumen; Tendenzen zu geringeren Jahrringbreiten geschädigter Bäume und dadurch bei Nadelhölzern zu größerem relativen Spätholzanteil und höherer Dichte.

Splintcharakteristika und Wassergehalt des Holzes: mehrere Untersuchungen in Deutschland und in der Schweiz zeigen, daß zwischen bestimmten Splintmerkmalen - vor allem dem flächenmäßigen Splintanteil - und dem äußerlich sichtbaren Gesundheitszustand von Nadelbäumen (besonders Fichte und Tanne) ein gesicherter Zusammenhang besteht: Geschädigte bzw. wenig vitale Bäume haben zumeist einen reduzierten Splintanteil, dessen Holz einen geringeren Wassergehalt aufweist als das gesunder Bäume. Hieraus folgt, daß die Schäden der Bäume und ihre Wasserversorgung zusammenhängen.

Mechanisch-technologische Eigenschaften: keinerlei Beeinträchtigungen; Festigkeit bei geschädigten Bäumen - wegen oft etwas höherer Rohdichte - tendenziell leicht höher als bei gesunden.

Biologische Resistenz (gegen holzverfärbende Organismen, Pilze und Insekten): kein Unterschied, sofern das Holz geschädigter Bäume sachgerecht geschlagen, gelagert und weiter verarbeitet wird (was ohnehin erforderlich ist). 
Übrige Gebrauchseigenschaften: keine Beeinträchtigung bekannt, außer verminderter Tränktiefe bei Rundholzimprägnierung von Bäumen mit reduzierter Splintbreite.

\section{Untersuchungsumfang, Material und Methoden}

Im Hinblick auf die mittlerweile recht zahlreichen - vor allem bundesdeutschen - Untersuchungen zur Holzqualität geschädigter Bäume war eine sorgfältige Abstimmung mit den bisherigen und laufenden Aktivitäten anderer Institute erforderlich. Von besonderem Interesse waren die Kenntnisse über die Holzqualität geschädigter und gesunder Bäume an alpinen Standorten. Darüber hinaus wurde angestrebt, solche Holzeigenschaften in die Untersuchung einzubeziehen, die in anderen Vorhaben nicht oder weniger berücksichtigt worden waren.

Auf dieser Basis wurde der in Tabelle 1 dargestellte Umfang der Untersuchung festgelegt. Aus zeitlichen und personellen Gründen war es unumgänglich, die sehr aufwendigen Prüfungen der verschiedenen Holzeigenschaften auf die wichtigsten Schweizer Nadelholzarten, Fichte und Weißtanne, zu beschränken. Für den Untersuchungsschwerpunkt wurden je 10 gesunde und kranke Fichten und Tannen von 4 charakteristischen Schweizer Standorten ausgewählt; von zwei weiteren Standorten wurden insgesamt 73 Bäume für Zusatzuntersuchungen entnommen.

Methodisch wurde die Arbeit als Vergleichsuntersuchung der verschiedenen Eigenschaften der beiden Kollektive „gesunde Bäume" und „geschädigte Bäume“ angelegt.

Zur Einschränkung des Versuchsaufwandes wurden einzelne Eigenschaften bzw. Eigenschaftsbereiche nur an Bäumen von einem oder zwei Standorten untersucht.

Bei der Auswahl der Probenstandorte wurden folgende schweizerische Waldregionen berücksichtigt: Alpen (2 Standorte), Voralpen, Mittelland (nordschweizerische Ebene, 2 Standorte) und Jorat-Colline (westschweizerische Hügelzone). Die Auswahl der Probebäume erfolgte - unter Beratung durch örtliche Forstleute - im Hinblick auf möglichst deutliche Unterschiede der Baumvitalität, beurteilt nach Nadelverlust und Kronenbild.

Es wurde darauf geachtet, daß sich die beiden Kollektive „gesunde Bäume“ und „geschädigte Bäume“ jedes Standorts nicht noch in anderer Hinsicht unterschieden, z. B. in der sozialen Stellung oder im durchschnittlichen Baumalter. Auf

Tabelle 1. Vergleichende Prüfung der Holzeigenschaften gesunder und kranker Fichten und Tannen in 5 Bereichen

\begin{tabular}{|c|c|}
\hline Gefügemerkmale & $\begin{array}{l}\text { Splintcharakteristika } \\
\text { Jahrringbreiten } \\
\text { Zellwandanteil } \\
\text { Rohdichte und Dichteverteilung }\end{array}$ \\
\hline $\begin{array}{l}\text { Feuchtephysikalische } \\
\text { Eigenschaften }\end{array}$ & $\begin{array}{l}\text { Wasserdampfsorption } \\
\text { Schwinden und Quellen } \\
\text { Kapillare Saugfähigkeit für Wasser }\end{array}$ \\
\hline $\begin{array}{l}\text { Mechanisch- } \\
\text { technologische } \\
\text { Eigenschaften }\end{array}$ & $\begin{array}{l}\text { Biegefestigkeit und Biege-E-Modul } \\
\text { Bruchschlagarbeit } \\
\text { Druckscherfestigkeit }\end{array}$ \\
\hline Biologische Resistenz & Fraßaktivität von Hausbock-Eilarven \\
\hline $\begin{array}{l}\text { Ausgewählte } \\
\text { Verarbeitungs- } \\
\text { eigenschaften }\end{array}$ & $\begin{array}{l}\text { Leimfugenfestigkeit } \\
\text { Verhalten gegenüber lasierender Ober- } \\
\text { flächenbehandlung } \\
\text { Verwitterungsverhalten von unbehandeltem } \\
\text { und lasierend gestrichenem Holz } \\
\text { Tränkbarkeit des Holzes bei Kesseldruck- } \\
\text { imprägnierung }\end{array}$ \\
\hline
\end{tabular}

diese Weise sollte eine allenfalls unterschiedliche Holzqualität nur durch den unterschiedlichen Gesundheitszustand erklärbar sein.

Allerdings ließ sich dieses Ziel nicht auf allen Standorten und oft nicht in der gewünschten Eindeutigkeit erreichen. Vielmehr ergaben sich bei der Auswahl der Probebäume häufig folgende Probleme:

Auf einzelnen Standorten war es nicht möglich, die gewünschte Anzahl vergleichbarer Fichten und Tannen mit starkem Nadelverlust (Schadensklasse "stark geschädigt“ bzw. über 65\% Nadelverlust gemäß Sanasilva-Klassierungsrichtlinien von 1985) zu finden. Deshalb mußten teilweise Bäume geringeren Schädigungsgrades einbezogen werden (mittelstark geschädigt, 30 bis $60 \%$ Nadelverlust). Auf der anderen Seite war es vereinzelt erforderlich, in das Kollektiv gesunde Bäume auch Bäume der Schadensklasse schwach geschädigt, bis $15 \%$ Nadelverlust, aufzunehmen.

Es wurde beobachtet (auf einzelnen Standorten auch als statistisch gesichert festgestellt), daß geschädigte Bäume tendenziell häufiger in sozial beherrschter bis unterdrückter als in herrschender Stellung standen. Außerdem bestand auf einzelnen Standorten ein gewisser Trend, daß die geschädigten Bäume durchschnittlich etwas älter waren als die gesunden. Da sich soziale Stellung und Baumalter auf die Vitalität der Bäume und somit auf deren Zuwachsleistung, Jahrringbreite und Holzdichte auswirken, konnten diese Parameter die möglichen Eigenschaftsunterschiede der Kollektive gesunde und geschädigte Bäume überlagern und vermischen.

Aus diesen Gründen kann davon ausgegangen werden, daß die ausgewählten Probebäume bzw. deren Holzqualität nicht repräsentativ für den jeweiligen Standort, geschweige denn für den Schweizer Wald waren (von der hierfür zu geringen Anzahl von Probebäumen abgesehen). Bei der allgemeinen Wertung der Versuchsergebnisse muß dieser Gesichtspunkt berücksichtigt werden.

Nach dem Fällen wurden jedem Baum je ein Probestück von 1 Meter Länge und eine Querschnittsscheibe von rd. $10 \mathrm{~cm}$ Länge (Dicke) aus dem unteren Stammabschnitt (Erdstamm) und dem oberen Stammabschnitt (Wipfelbereich) entnommen. Außerdem wurden die frischen Schnittflächen des Erdstammes fotografiert, insbesondere im Hinblick auf die Bestimmung des flächenmäßigen Splintanteils mit dem Bildanalyseautomaten.

Unmittelbar nach Entnahme wurden die Proben - insbesondere die Querschnittscheiben - in der EMPA weiterbearbeitet, um die Holzfeuchte im Zeitpunkt der Baumfällung möglichst genau ermitteln zu können. Die Proben wurden ausgeformt und, soweit erforderlich, im Normklima von $20^{\circ} \mathrm{C} / 65 \%$ r.F. bis zu ihrer Prüfung auskonditioniert. Die nicht zu konditionierenden Versuchskörper verblieben in einem klimatisierten Holzlager. Alle Proben für die Festigkeitsprüfungen an strukturstörungsfreiem $\mathrm{Holz}$ wurden aus gespaltenen Rohlingen hergestellt, um die notwendige Probenqualität zu erzielen (Ausrichtung auf Jahrringstellung; Probenachsen faserparallel). Einzelheiten der Untersuchungsmethoden siehe Sell et al. (1988).

\section{Ergebnisse}

\subsection{Splintanteil}

(je 10 gesunde und kranke Fichten und Tannen von 4 Standorten)

Der Splintanteil der kranken Bäume war erheblich geringer als der gesunder Vergleichsbäume. Das Defizit der durchschnittlichen absoluten Splintbreite betrug bei kranken Fichten rd. 30\% im unteren Stammabschnitt und 40\% im Wip- 
felbereich; die entsprechenden Werte bei Weißtannen sind rd. $40 \%$ und $20 \%$. Bei den kranken Fichten waren die Splintanteile auf dem alpinen und voralpinen Standort besonders gering, auf dem Mittelland-Standort am wenigsten vermindert. Kranke Tannen wiesen auf allen Standorten im unteren Stammbereich einen etwa gleich verminderten Splintanteil auf; im Wipfelbereich war die Verminderung auf dem voralpinen Standort signifikant größer als anderswo. Der Wassergehalt des Splints gesunder und kranker Fichten und Tannen war nur geringfügig und meist nicht signifikant verschieden. Aufgrund dieses Befundes kann festgestellt werden, daß der nach dem Benadelungsbild beurteilte Gesundheitszustand der Bäume mit ihrem Wasserhaushalt zusammenhängt.

\subsection{Jahrringbreite}

(je 10 gesunde und kranke Fichten und Tannen von 4 Standorten)

Die gesunden Bäume haben im Mittel breitere Jahrringe ausgebildet als die erkrankten (Bilder 1 u. 2). Dieser Unterschied ist bei den Fichten an allen, bei den Tannen nur an den Alpen- und Voralpenstandorten ausgeprägt. Die festgestellten Unterschiede der Vitalität von gesunden und geschädigten Bäumen sind offensichtlich zu einem erheblichen Teil durch ihre soziale Stellung im Bestand verursacht. Die langfristigen Abnahmen der Jahrringbreiten bei beiden Baumarten, insbesondere in den Alpen und Voralpen, haben altersbedingte Ursachen. Seit etwa 1960 fällt ein ungewöhnlich starker Abfall des Jahrringverlaufs lediglich bei den Tannen des Mittellandes auf, und zwar sowohl bei den gesunden wie bei den geschädigten Bäumen. Hier deutet auch ein mehrfach festgestellter frühzeitiger Stillstand des Jahrringwachstums auf einen (unbekannten) Schadeinfluß hin. Allerdings ist hier seit 1980 wieder eine starke Zunahme der Jahrringbreite mehrerer Bäume feststellbar; deutliche Zuwachszunahmen während der letzten rd. 10 Jahre wurden auch bei den Tannen der anderen Standorte beobachtet, nicht hingegen bei den Fichten.

\subsection{Zellwandanteil}

(je 10 gesunde und kranke Fichten von einem Standort)

Die radiale Verteilung des prozentualen Zellwandanteils und damit der Rohdichte des Holzes gesunder und geschädigter Fichten eines Mittelland-Standortes zeigt für den untersuchten Zeitraum von 1950 bis 1985 keinen abnehmenden Trend. Im Hinblick auf den mittleren Zellwandanteil bestehen zudem zwischen den gesunden und geschädigten MittellandFichten keine Unterschiede.
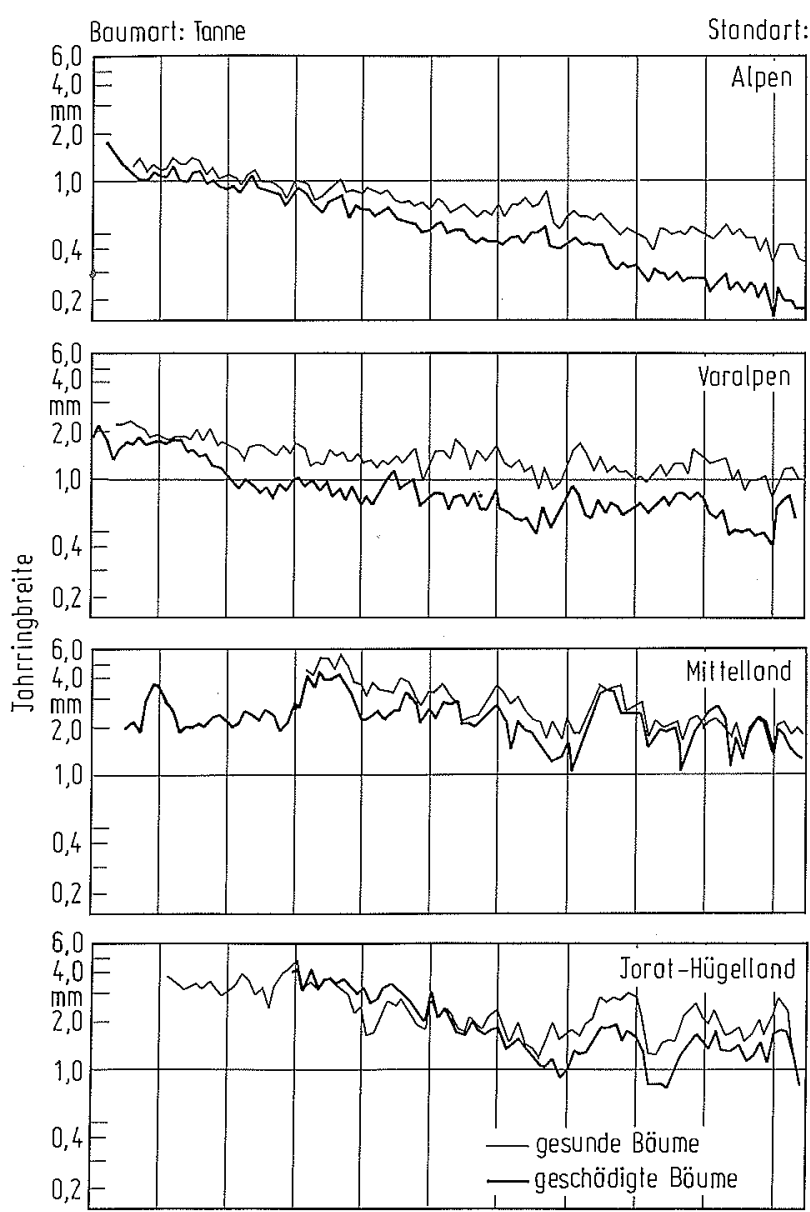

$\begin{array}{lllllllllll}1880 & 1890 & 1900 & 1910 & 1920 & 1930 & 1940 & 1950 & 1960 & 1970 & 1980\end{array}$

Bild 1. Mittelkurven der Jahrringbreiten von je 10 gesunden und kranken Fichten von 4 Standorten

Fig. 1. Average chronologies of the growth ring width of 10 healthy and diseased spruce trees of 4 Swiss locations
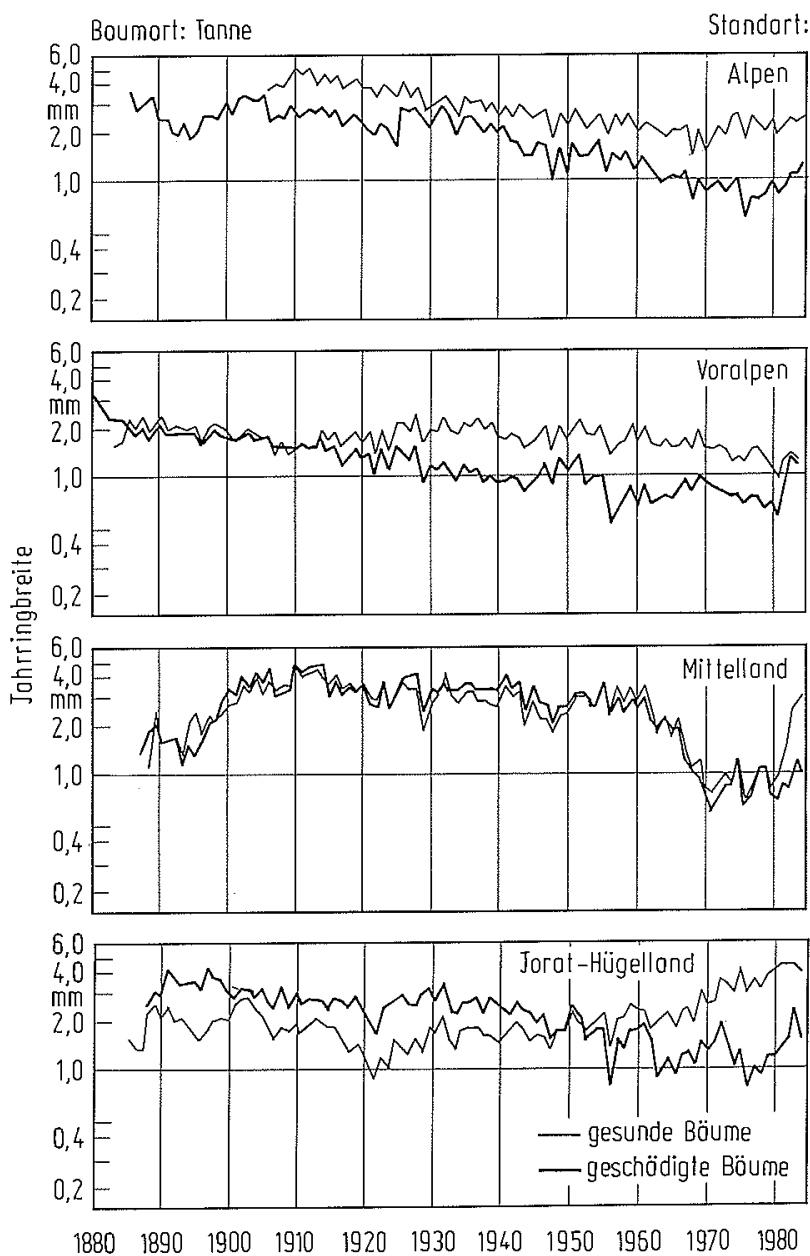

Bild 2. Mittelkurven der Jahrringbreiten von je 10 gesunden und kranken Tannen von 4 Standorten

Fig. 2. Average chronologies of the growth ring width of 10 healthy and diseased white firs of 4 Swiss locations 
4.4 Holzfeuchte- und Dimensionsänderungen (je 10 gesunde und kranke Fichten und Tannen von 2 Standorten)

Bei Änderungen der relativen Luftfeuchte des Umgebungsklimas zeigt Holz von gesunden und geschädigten Fichten und Tannen praktisch identische Holzfeuchte- und Dimensionsänderungen. Die Gleichgewichtsholzfeuchten und Quellwerte stimmen zudem sehr gut mit bekannten Daten dieser Hölzer überein. Geringfügig höhere Quellwerte von feinjährigem und darum etwas dichterem Holz entsprechen ebenfalls der bestehenden Kenntnis.

\subsection{Kapillare Saugfähigkeit in Faserrichtung} (je 10 gesunde und kranke Fichten und Tannen von 4 Standorten)

Die sehr heterogenen Ergebnisse des Saugfähigkeitsversuchs mit Wasser bei Normaldruck sind - insbesondere bei der Tanne - nicht einfach zu interpretieren. Es ist zu vermuten, daß vor allem beim Tannen-Kernholz, wo die größten Streuungen der kapillaren Wasseraufnahme beobachtet wurden, Gefügeunterschiede bestehen, insbesondere in der Hoftüpfelanatomie. Bemerkenswert ist, daß hohe kapillare Wasseraufnahmen bei Tannenproben mit und ohne Naßkern festgestellt wurden. Im Durchschnitt zeigte sich aber, wie zu erwarten war, daß die kapillare Saugfähigkeit der Weißtanne erheblich größer ist als bei der Fichte, nämlich um rd. $65 \%$ beim Splintholz und um fast den Faktor 3 beim Kernholz; vgl. Bild 3. Mit der Vitalität der Bäume zusammenhängende Unterschiede der Saugfähigkeit wurden weder bei Tanne noch bei Fichte festgestellt.

\subsection{Biegefestigkeit und $E$-Modul,}

kleine störungsfreie Proben

(je rd. 10 gesunde und kranke Fichten und Tannen von 2 Standorten)

Die Ergebnisse der Biegeprüfungen entsprechen - unter Berücksichtigung der jeweiligen Holzdichte und der Literaturangaben - den Erwartungen. Biegefestigkeit und E-Modul des Holzes geschädigter Bäume sind nicht geringer als bei gesunden Bäumen, sondern tendenziell sogar etwas höher (Bild 4). Dies hängt offensichtlich auch damit zusammen,
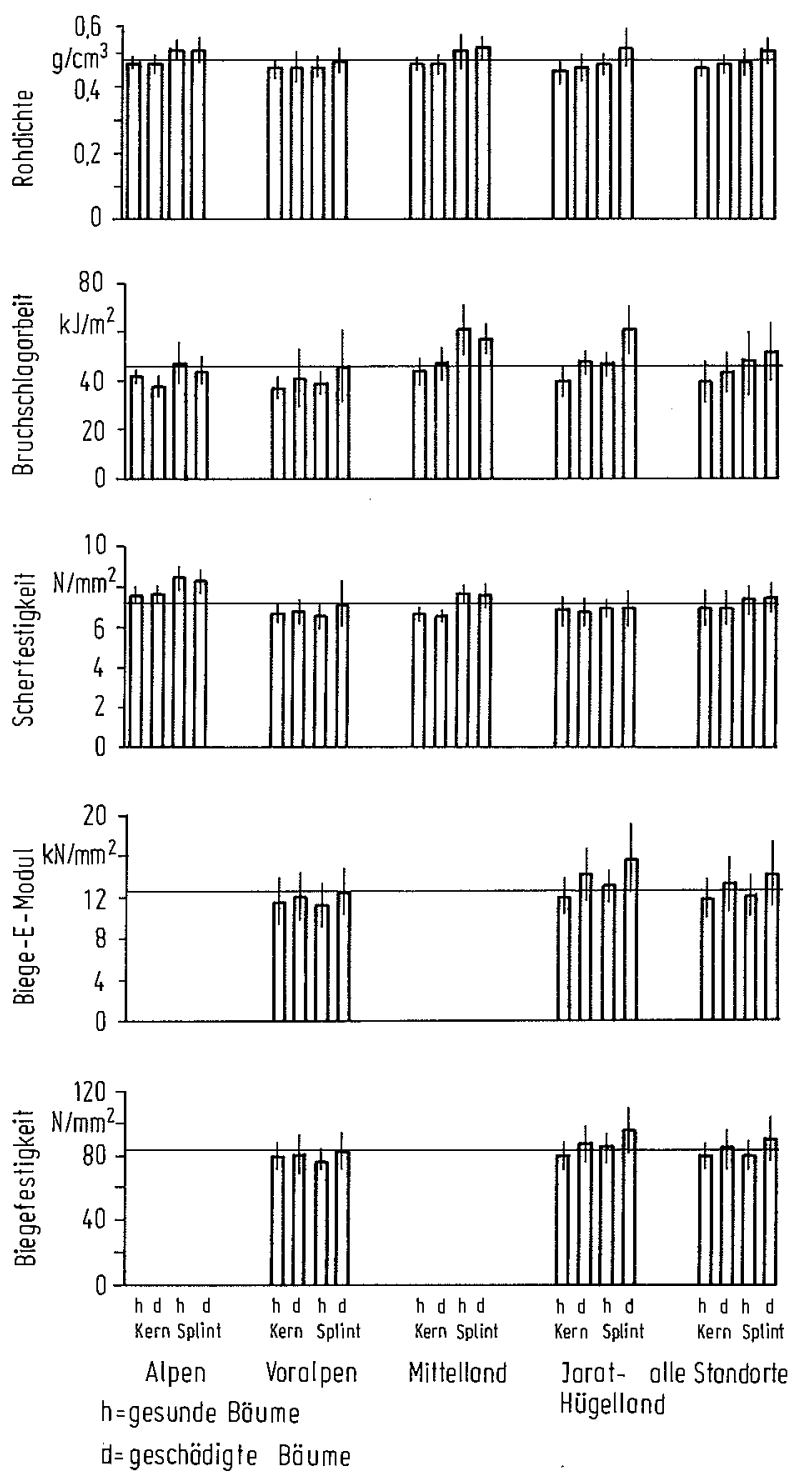

Bild 4. Rohdichte und Festigkeitseigenschaften von Splint- und Kernholz von rd. 10 gesunden und kranken Fichten ( 4 bzw. 2 Standorte). Die Resultate bei Weißtanne sind sehr ähnlich

Fig. 4. Density and strength properties of the sapwood and heartwood of 10 healthy and diseased spruce trees ( 4 and 2 locations, respectively). The results are quite similar for white fir

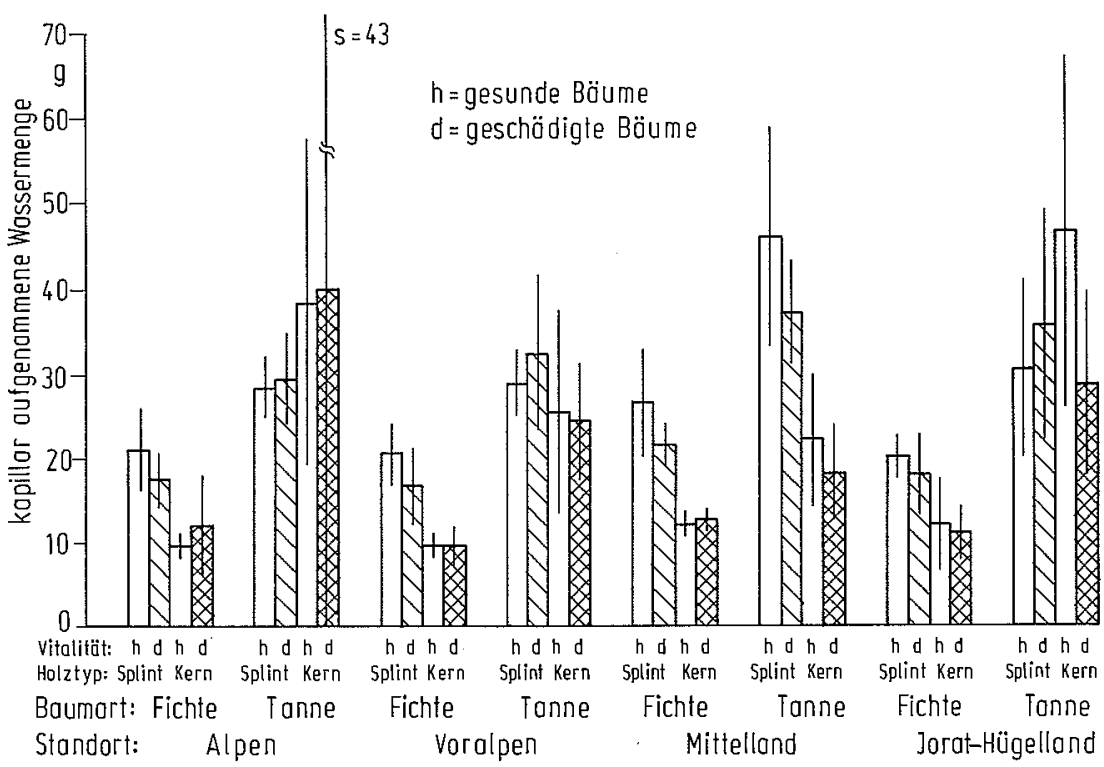

Bild 3. Kapillare Aufsaugung von Wasser in Faserrichtung von Fichten- und Tannenholz (je 10 gesunde und kranke Bäume, Splint und Kern, 4 Standorte) Fig. 3. Capillary absorption of water (in fiber direction) by wood of spruce and white fir (10 healthy and diseased trees each, sapwood and heartwood, 4 locations) 
daß die soziale Stellung und damit die Wachstumsbedingungen der geschädigten Bäume eines Standortes durchschnittlich eher schlechter waren als bei den gesunden Bäumen, was zu schmaleren Jahrringen (siehe dort) und somit zu etwas größeren Rohdichten und Festigkeiten der geschädigten Bäume führte.

\subsection{Biegefestigkeit und E-Modul, Balken in Bauholzdimension \\ (gesunde und kranke Fichten von einem Standort)}

Im Vergleich zu den Festigkeiten von kleinen störungsfreien Proben aus dem Holz gesunder und geschädigter Bäume sind die Unterschiede der Biegefestigkeiten und des Biege-E-Moduls der Balken normaler Bauholzqualität noch deutlicher. Die signifikant höheren Festigkeiten des Holzes von kranken Fichten liegen eindeutig in der höheren Rohdichte des engringigeren Holzes der untersuchten geschädigten Bäume begründet (Bild 5), die offensichtlich schon sehr frühzeitig (und nicht erst während der letzten Jahrzehnte) unter schlechteren Wachstumsbedingungen standen als die gesunden Bäume des gleichen Standorts.

\subsection{Bruchschlagarbeit}

(je 10 gesunde und kranke Fichten und Tannen von 4 Standorten)

Erwartungsgemäß zeigen die Ergebnisse der Schlagbiegeprüfung eine erhebliche Streuung und - sicherlich $z$. T. gerade deswegen - ein uneinheitliches Bild. Es kann aber eindeutig festgestellt werden, daß die Bruchschlagarbeit, die ein sehr empfindlicher Indikator selbst für kleine Gefügestörungen des Holzes ist, beim Holz der geschädigten Fichten und Tannen nicht geringer ist als bei den gesunden Bäumen (Bild 4). Im Gegenteil, es zeigt sich die schon bei der statischen Biegefestigkeit beobachtete Tendenz, daß die Bruchschlagarbeit beim Holz geschädigter Bäume mit seinen durchschnittlich etwas engeren Jahrringen etwas höher liegt; diese Unterschiede sind aber, vor allem wegen der großen Streuung der Messergebnisse, statistisch nicht gesichert.

\subsection{Scherfestigkeit}

(je 10 gesunde und kranke Fichten und Tannen von 4 Standorten)

Die sehr geringen Unterschiede der Scherfestigkeit zwischen den Fichten und Tannen der verschiedenen Standorte und insbesondere die praktisch gleichen Festigkeitswerte der beiden Probengruppen gesunde/geschädigte Bäume zeigen, daß die Festigkeit des Holzgefüges quer zur Faser bei geschädigten Bäumen weder im Splintholz noch im Kernholz beeinträchtigt ist. Gerade bei der Scherfestigkeit fällt im übrigen die bemerkenswerte Homogenität der Holzfestigkeit der untersuchten Bäume auf, die in deutlicher Übereinstimmung zu der einheitlich recht hohen Rohdichte des untersuchten Probenmaterials steht (Bild 4).

\subsection{Trocknungsverhalten}

Das Trocknungsverhalten des Holzes gesunder und geschädigter Bäume wurde experimentell nicht untersucht, sondern aus den Ergebnissen der feuchtephysikalischen Prüfungen abgeleitet. Abgesehen vom Splintanteil der frisch gefällten

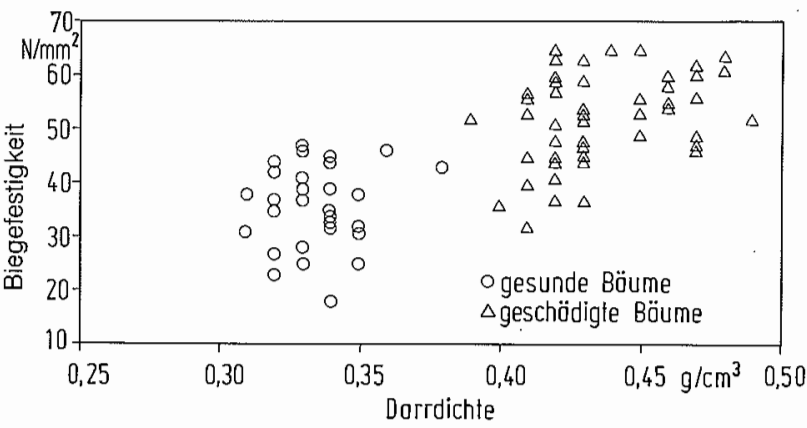

Bild 5. Zusammenhang zwischen Biegefestigkeit und Rohdichte von Balken normaler Bauholzqualität; 32 Proben aus 5 gesunden, 54 Proben aus 18 kranken Fichten, 1 Standort. Die kranken Bäume zeigen wegen ungünstigerer Wachstumsbedingungen eine wesentlich geringere Jahrringbreite, daher eine deutlich höhere Dichte und Biegefestigkeit, verglichen mit dem Holz gesunder Bäume

Fig. 5. Correlation between modulus of rupture and wood density of beams (normal structural timber), 32 samples from 5 healthy trees, 54 samples from 18 diseased trees, 1 location. The diseased trees exhibit a markedly reduced average growth ring width and, therefore, a significantly higher density and bending strength compared with wood of healthy trees. These differences are caused by different growth conditions

Probebäume bestehen bei den die Trocknung beeinflussenden Merkmalen (Wassergehalt des Holzes, Sorptionsverhalten, kapillare Saugfähigkeit) zwischen gesunden und geschädigten Bäumen keine statistisch gesicherten Unterschiede, aus denen sich ein unterschiedliches Trocknungsverhalten ableiten ließe. Auch der Tannennaßkern, der bekanntlich wegen seines hohen Wassergehalts relativ lange Trockungszeiten erfordert, trat bei den geschädigten Bäumen aller Standorte nicht häufiger auf als bei den gesunden.

\subsection{Scherfestigkeit der Leimverbindungen (je rd. 10 gesunde und kranke Fichten und Tannen von 2 Standorten)}

Die Prüfung der Scherfestigkeit der Leimverbindungen von Holzproben erbrachte keine Unterschiede der Verleimungsgüte des Holzes gesunder und geschädigter Bäume. Andere Unterschiede zwischen Probenkollektiven, insbesondere die höhere Leimfugenfestigkeit der Tannenproben gegenüber den Fichtenproben, entsprechen den Erwartungen und lassen sich zumindest zu einem Teil durch die Gefügestruktur der Hölzer erklären, vor allem durch die größere kapillare Saugfähigkeit der Tanne gegenüber der Fichte, die zu einer etwas besseren Verankerung des Leims beim Tannenholz führt.

\subsection{Verhalten bei Bewitterung; anstrichtechnische Merkmale \\ (je 10 Fichten- und Tannenproben gesunder und kranker Bäume von 2 Standorten)}

Wie aufgrund vorangegangener Teiluntersuchungen zu erwarten war, unterscheidet sich das Holz gesunder und geschädigter Bäume auch im Hinblick auf die komplexen Eigenschaften „Verhalten bei Bewitterung, anstrichtechnische Merkmale" nicht. Einer Verwendung des Holzes geschädigter Bäume für Bereiche mit direkter Wetterbeansspruchung (Fassadenverkleidungen, Balkone, Fenster usw.) steht daher auch aus dieser Sicht nichts entgegen. Recht bedeutsam scheint in diesem Zusammenhang, daß Bläuepilzbefall und 
Rotstreifigkeit infolge längerer Lagerung von waldfrischen Stammabschnitten beim Holz geschädigter Bäume nicht intensiver waren als bei den gesunden Bäumen.

\subsection{Imprägniertiefe}

(je 20 Fichten und je 5 Tannen der Vitalitätsstufen gesund bzw. absterbend, von einem Standort)

Die großen Streuungen der Meßdaten der Imprägniertiefe innerhalb der Kollektive sind durch die Individualität des Baumes, seine soziale Stellung, das Mikroklima und die Bodenbeschaffenheit begründet. Hinzu kommt, daß der Benadelungszustand eines Baumes nicht immer mit dem Gesundheitszustand des Stammes übereinstimmte. Es zeigte sich nämlich teilweise, daß absterbende Bäume einen zumindest optisch gesunden Querschnitt aufwiesen, während als gesund taxierte Bäume im unteren Erdstammbereich $u$. U. be-
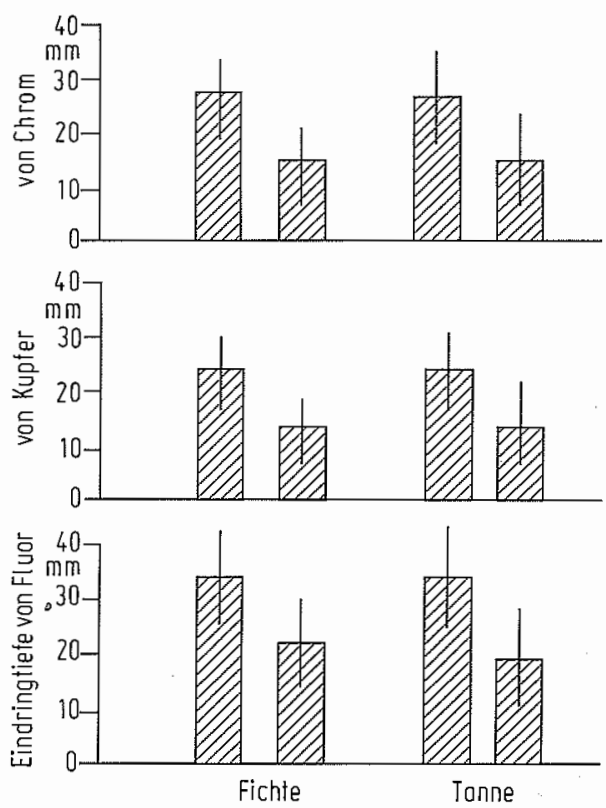

Bild 6. Radiale Eindringtiefe der Komponenten einer CFK-Salzlösung in Rundholzproben von gesunden und absterbenden Fichten (je 20 Bäume) und Tannen (je 5 Bäume); Gesamtmittelwerte und mittlere maximale sowie minimale Eindringung. Linke Säule: gesund; rechte Säule: absterbend

Fig. 6. Penetration depth of the compounds of a CCF-preservative solution into round wood samples of healthy and dying spruces (20 trees each) and white firs ( 5 trees each), 1 location; total averages and average maximum and minimum penetration. Left column: sound; right column: diseased reits Braunverfärbungen und Weißfäule zeigten. Jedenfalls wurde die Tränkbarkeit des saftfrischen Rundholzes im Wechseldruckverfahren durch die Vitalität des Baumes beeinflußt (Bild 6). Während bei der Fichte die Eindringtiefe der Schutzsalze wohl primär mit der Verringerung des Splintanteils geschädigter Bäume abnimmt, schienen bei der Tanne noch weitere Faktoren von Bedeutung zu sein: Obwohl die Feuchteunterschiede im Splint, gesunder" und „absterbender" Tannen geringer waren als bei den Fichten, war doch eine ähnlich große Reduktion der Eindringtiefe der Schutzsalze der imprägnierten Querschnittfläche von Stammabschnitten bei den geschädigten Tannen zu verzeichnen wie bei den Fichten.

\subsection{Resistenz gegenüber Hausbocklarven}

Trockenes Holz von Fichte weist gegenüber dem Hausbock eine höhere Resistenz auf als das von Tanne aus dem gleichen Wuchsgebiet. Die besonders bei den Fichten beobachtete größere Hausbock-Resistenz des Holzes geschädigter gegenüber gesunden Bäumen dürfte zumindest teilweise eine Folge eines veränderten Nährstoffangebotes sein: Je geringer die assimilierende Nadelmasse eines Baumes ist, um so weniger Nähr- und Speicherstoffe können assimiliert und im Holz eingelagert werden; dementsprechend steht der Hausbocklarve ein geringeres Nährstoffangebot zur Verfügung.

\section{Literatur}

Schrader, S.; Greve, U.; Schönwald, H. R. 1983: Saure Niederschläge und Waldschäden, Bibliographie. Mitt. Bundesforsch. Anst. Forst- u. Holzwirtsch. (BFH), Hamburg, Nr. 138

Schrader, S.; Schönwald, H. R.; Greve, U. 1984: Immissionen und Waldschäđen, Bibliographie. Mitt. Bundesforsch. Anst. Forstu. Holzwirtschaft (BFH), Hamburg, Nr. 145

Schrader, S.; Schönwald, H. R.; Dujesiefken, D. 1985: Immissionen und Waldschäden. Bibliographie III, 1984. Mitt. Bundesforsch. Anst. Forst- u. Holzwirtschaft, Hamburg, Nr.149

Schrader, S.; Schönwald, H. R.; Dujesiefken, D. 1986: Immissionen und Waldschäden. Bibliographie IV, 1985. Mitt. Bundesforsch. Anst. Forst- u. Holzwirtschaft, Hamburg, Nr. 153

Schulz, H.; Aufsess, H. v.; Bues, C. T.; Fengel, D.; Grosser, D. 1988 Eine Abhängigkeit von Holzqualität und Waldschäden besteht nicht. Holz-Zbl. 144:854-859

Sell, J.; Schnell, G. R.; Arnold, M.; Graf, E.; Richter, K. 1988: Holzqualität gesunder und geschädigter Fichten und Tannen von 6 schweizerischen Standorten. EMPA-Bericht Nr.217, Dübendorf/Schweiz, $112 \mathrm{~S}$. 